\title{
Conocimiento didáctico-matemático en docentes de básica y media
}

\section{Didactic-mathematical knowledge in basic and media teachers}

Raúl Prada Núñez ${ }^{1}$

Audin Aloiso Gamboa Suárez ${ }^{2}$

Cesar Augusto Hernández Suárez ${ }^{3}$

UFPS

\section{RESUMEN}

El presente artículo de investigación muestra un análisis cuantitativo - descriptivo de las características de un grupo de docentes en ejercicio que orientan la asignatura de Matemáticas en diversas instituciones educativas de carácter oficial en la ciudad de San José de Cúcuta - Colombia. Se aplicó un cuetionario a 73 docentes de 13 instituciones públicas aplicando para su selección el muestreo no probabilístico bajo la aplicación de la técnica de muestreo intencional. Los resultados 1 Docente investigador de la Universidad Francisco de Paula Santander. Magíster en Ingeniería de Análisis de Datos, Mejora de Procesos y Toma de Decisiones por la Universidad Politécnica de Valencia (España). Correo electrónico: raulprada@ufps.edu.co, Orcid: orcid.org/00000001-6145-1786.

2 Docente investigador de la Universidad Francisco de Paula Santander. Doctor en Ciencias de la Educación por la Universidad de Cartagena. Correo electrónico: audingamboa@ufps.edu.co, Orcid: orcid.org/0000-0001-9755-6408. 3 Docente investigador de la Universidad Francisco de Paula Santander. Magíster en Enseñanza de las Ciencias por la Universidad Nacional Experimental del Estado Táchira (Venezuela). Correo electrónico: cesaraugusto@ ufps.edu.co, Orcid: orcid.org/0000-0002-7974-5560. muestran que las concepciones y conocimientos su actuar pedagógico y se relacionan con la preparación de las clases, las actividades en las aulas, los recursos didácticos que implementa en el proceso de enseñanza y la evaluación de los aprendizajes.

PALABRAS CLAVE: didáctica; enseñanza de la matemática; conocimiento del maestro; educación básica.

\section{ABSTRACT}

This research article shows a quantitativedescriptive analysis of the characteristics of a group of practicing teachers who guide the subject of Mathematics in various official educational institutions in the city of San José de Cúcuta - Colombia. A questionnaire was applied to 73 teachers from 13 public institutions applying non-probabilistic sampling for their 
selection under the application of the intentional sampling technique. The results show that the mathematical conceptions and knowledge that the teachers have guide their pedagogical actions and are related to the preparation of the classes, the activities in the classrooms, the didactic resources that they implement in the teaching process and the evaluation of learning.

KEYWORDS: didactics; teaching of mathematics; knowledge of the teacher; basic education

\section{INTRODUCCIÓN}

En las últimas tres décadas ha sido innegable la importancia que ha cobrado para la Educación Matemática el analizar al docente en ejercicio de su profesión, dada la importancia que tiene su rol en el proceso de aprendizaje de los estudiantes. En este sentido se han desarrollado diversas investigaciones en las que se reconoce su influencia tal como lo reportan los trabajos de Gómez-Chacón, Op’t Eynde \& De Corte (2006), Mayorca, Camacho, Trujillo y Artunduaga (2009), Gamboa $(2014,2016)$ y Escobar (2015), entre otros.

Un campo de interés en Educación Matemática corresponde a la gran diversidad de propuestas existentes sobre posibles modelos o categorizaciones que intentan identificar los aspectos o características que debe poseer un docente de Matemáticas para poder garantizar el objetivo perseguido en el proceso educativo, que no es más que el estudiante entienda y aplique los conceptos matemáticos en la búsqueda de soluciones a los problemas que les ofrece su cotidianidad.

Con esta investigación se pretender determinar el tipo de conocimiento didáctico-matemático que poseen un grupo de docentes en ejercicio que orientan la asignatura de Matemáticas en diversas instituciones educativas de carácter público del Departamento Norte de Santander.

\section{MODELOS DEL CONOCIMIENTO MATEMÁTICO QUE POSEE EL DOCENTE}

En la Tabla 1 se presenta una síntesis del trabajo adelantando por Pino y Godino (2015) quienes realizan un ensayo teórico sobre la diversidad de modelos existentes para caracterizar los conocimientos del profesorado de Matemáticas, en el que se discuten los hallazgos y aportes de cada uno de ellos. Si el lector está interesado en profundizar en la explicación de cada una de las dimensiones de clasificación se recomienda consultar dicho documento. 
Tabla 1. Modelos de conocimiento del profesor de Matemáticas

\begin{tabular}{cl}
\hline \multicolumn{1}{c}{ Autor } & \multicolumn{1}{c}{ Clasificación } \\
\hline \multirow{3}{*}{ Shulman (1986) } & Conocimiento del Contenido \\
& Conocimiento Pedagógico del Contenido \\
& Conocimiento Curricular \\
\hline & Conocimiento del Contenido \\
& Conocimiento Pedagógico General \\
& Conocimiento Curricular \\
& Conocimiento Pedagógico del Contenido \\
& Conocimiento de los Estudiantes y de sus características \\
& Conocimiento de los Contextos Educativos \\
& Conocimiento de los fines, propósitos y valores de la educación \\
& Conocimiento Pedagógico General \\
& Conocimiento del Contenido \\
& Conocimiento Pedagógico del Contenido \\
& Concepciones de las propuestas para la enseñanza de un contenido \\
& Conocimiento de la Comprensión de los estudiantes \\
& Conocimiento Curricular \\
& Conocimiento de las Estrategias Institucionales \\
& Conocimiento del Contexto \\
\hline Grossman (1990) & Conocimiento del Contenido \\
& Conocimiento Especializado del Contenido \\
& Conocimiento Común del Contenido \\
Cill, Ball \& Schilling (2008) & $\begin{array}{l}\text { Conocimiento en el Horizonte Matemático } \\
\text { Conocimiento Pedagógico del Contenido } \\
\text { Conocimiento del Currículo } \\
\text { Conocimiento del Contenido y los Estándares } \\
\text { Conocimiento del Contenido y la Enseñanza }\end{array}$ \\
\hline
\end{tabular}

Como se puede observar en las clasificaciones sugeridas por los autores, se evidencian como recurrentes el conocimiento del contenido y el conocimiento pedagógico del contenido, con lo que queda claro es que todo docente de Matemáticas debe ser competente no sólo en su saber disciplinar, sino que debe poseer competencias pedagógicas que faciliten el proceso de aprendizaje.

\section{METODOLOGÍA}

Esta investigación se realizó con una población finita equivalente a la totalidad de docentes de 13 instituciones educativas de carácter público con domicilio en la ciudad de San José de Cúcuta vinculados laboralmente con ellas. La vinculación de las instituciones educativas fue mediante muestreo no probabilístico bajo la aplicación de la técnica de muestreo intencional, dado que se realizó acercamiento a 25 instituciones educativas y de ellas sólo aceptaron ser parte de la investigación el número ya mencionado. En un segundo momento del muestreo se define como criterio de inclusión el orientar la asignatura de Matemáticas entre los grados de Sexto a Undécimo, logrando completar un tamaño de muestra de 73 docentes.

Se diseñó un cuestionario con cuatro secciones distribuidos así: a) Primera sección correspondiente al registro de la información 
general del docente asociado con su perfil demográfico con 7 ítems todos con opciones de respuesta cerradas; b) Segunda sección en la que se incorporan una serie de ítems tendientes a caracterizar el sistema de creencias que poseen los docentes sobre las Matemáticas y su proceso de enseñanza con 42 ítems valorados mediante escala Likert de cinco niveles; c) Tercera sección se listan una serie de aspectos propios de las competencias matemáticas que se deben promocionar en el aula para garantizar la correcta apropiación de los conceptos matemáticos con 49 ítems evaluados mediante escala Likert con cinco niveles de frecuencia de ocurrencia; d) Cuarta sección correspondiente a las características de la práctica pedagógica que desarrolla el docente con 34 ítems valorados también con una escala Likert de frecuencia de ocurrencia de eventos.

Elcuestionariofue diligenciadoen cada institución educativa mediante cita concertada con el docente durante el año 2019. Posteriormente los datos fueron registrados en una base de datos mediante el uso del software SPSS v25, para ser analizados descriptivamente y poder generar al final una serie de perfiles docentes en función de los ítems considerados mediante la aplicación del análisis factorial exploratorio AFE, previa validación de la escala utilizada.

A partir de las características mencionadas se concluye que esta investigación adopta un enfoque cuantitativo a nivel descriptivo.

\section{RESULTADOS Y DISCUSIÓN}

\section{Características demográficas}

En cuanto a las características demográficas de los docentes encuestados se determinó predominio del sexo femenino en la muestra, es decir, que las mujeres se han inclinado por el estudio de esta disciplina que inicialmente era considerada para los hombres, tal como lo reseñan Nortes y Nortes (2017) quienes concluyen que "a los hombres les agradan las matemáticas más que a las mujeres, aunque ambos las consideran útiles por igual" (p. 27). En esta línea argumentativa en el trabajo de Márquez y Ramos (2018) se cuestiona la creencia de que las mujeres no eran talentosas para las Matemáticas, en este sentido las autoras concluyen que "el problema no está en las mujeres, sino en la forma de concebir al conocimiento y a quien es capaz de adquirirlo" (Márquez y Ramos, 2018, p.34).

Continuando con la descripción se identificó que aproximadamente el $85 \%$ de los docentes que orientan la asignatura de Matemáticas, tienen formación pedagógica de pregrado con énfasis en la disciplina. Arce, Alpízar, López, Ramírez y Huertas (2013) resaltan que "la calidad de la educación está relacionada con la eficacia de la enseñanza que ofrece el docente" (p. 1) y esta depende tanto de la formación inicial como de la formación continua, es decir, que la actividad docente demanda de un proceso permanente de actualización en el que invite al docente (independientemente de su formación de pregrado) a hacer de su trabajo una actividad reflexiva y crítica.

Esta capacitación permanente los docentes la realizan de dos formas, mediante cursos cortos de actualización o por medio de la realización de programas de posgrado. En este sentido se determinó que aproximadamente el $55 \%$ de los docentes encuestados habían cursado algún estudio de posgrado con predominio a nivel de especialización. Este nivel de formación posgradual, aporta conocimientos específicos en un área en particular pero sin mucho énfasis en el desarrollo de competencias investigativas, siendo este un atractivo para los docentes, puesto que como se afirma en Hernández (2009) una de las principales dificultades que experimentan los estudiantes al nivel de maestría, corresponde a la realización de la investigación y posterior escritura académica o 
llamada también alfabetización académica que les impide culminar sus trabajos de grado.

En la investigación realizada por Echeverría et al. (2017) se resalta que en la actualidad en los diversos programas de postgrado están interactuando al menos tres generaciones de personas (Baby Boomers, Generación $X$ y Generación $Y$ o Milenials) que, para el caso de los integrantes de esta muestra, se tiene que el $50.7 \%$ de ellos pertenecen generacionalmente a las dos primeras de ellas, es decir, que los docentes tiene más de 40 años de edad. Este aspecto es muy importante puesto que existen notorias diferencias entre las herramientas didácticas y tecnológicas implementadas en el proceso educativo de una generación a otra. En este sentido se puede afirmar que

... al docente en turno le cuesta el doble de esfuerzo aprender a utilizar las nuevas herramientas, romper sus propios paradigmas con el fin de poder dirigir una correcta utilización de las herramientas actuales a los educandos, dirigiendo y enseñando en dónde y cómo buscar información "correcta" en el mar de la información globalizada, la responsabilidad del docente. No sólo es tener el conocimiento de la asignatura y transmitirlo, si no ahora está la responsabilidad de aprender a utilizar las herramientas actuales (TIC), enseñar a los estudiantes a encontrar la información correcta en los sitios web correctos, analizar esta información y convertirla en competencias para su desarrollo; para poder lograr esto, los docentes deben ser actualizados con la visión que son hijos de Baby Boomers, son Generación $X$ y son padres y profesores de Milenials, actualmente los primeros Milenials ya también son profesores de milenials y padres de una nueva generación llamada "Generación Z" o XXL a quienes se les conoce como los verdaderos nativos digitales (Echeverría et al., 2017, p. 34).

Estas afirmaciones cobran importante relevancia, puesto que se evidencia que aproximadamente el $54.8 \%$ de los encuestados cuentan con once o más años de experiencia como docentes y de ellos el 32.9\% llevan dedicados más de veinte años a la actividad de enseñanza. Al parecer estos docentes fueron formados con unas metodologías y recursos que hoy día no funcionaran dadas las características de los estudiantes con los que se cuentan en la actualidad en las aulas. En este sentido se afirma que

Hoy día, se requiere que los docentes estén conscientes de las necesidades de aprendizaje de las nuevas generaciones del Siglo XXI, centradas en ellos, facilitándoles el aprendizaje autónomo y desarrollando competencias para la vida, atentos al cambio y la resolución de situaciones y problemas (Echeverría et al., 2017, p. 35).

Como se ha mencionado, el proceso de enseñanza en la contemporaneidad, debe estar centrado a motivar en los estudiantes el desarrollo del pensamiento crítico de tal forma que estos vean en los conocimientos que se abordan en la escuela, una herramienta de apoyo para dar solución a los problemas a los que se enfrentan a diario, independientemente si vive en un entorno rural o urbano. 
Tabla 2. Perfil demográfico de los encuestados

\begin{tabular}{|c|c|c|c|}
\hline Característica & Opciones de respuesta & Frecuencia & Porcentaje \\
\hline \multirow{3}{*}{ Sexo } & Femenino & 43 & 58.9 \\
\hline & Masculino & 30 & 41.1 \\
\hline & Total & 73 & 100.0 \\
\hline \multirow{3}{*}{ Formación profesional } & Licenciado(a) & 62 & 84.9 \\
\hline & Ingeniero(a) & 11 & 15.1 \\
\hline & Total & 73 & 100.0 \\
\hline \multirow{4}{*}{$\begin{array}{l}\text { Máximo nivel de posgrado } \\
\text { alcanzado }\end{array}$} & Especialización & 32 & 43.8 \\
\hline & Maestría & 8 & 11.0 \\
\hline & Ninguno & 33 & 45.2 \\
\hline & Total & 73 & 100.0 \\
\hline \multirow{5}{*}{ Edad } & De 20 a 30 años & 17 & 23.3 \\
\hline & De 31 a 40 años & 19 & 26.0 \\
\hline & De 41 a 50 años & 23 & 31.5 \\
\hline & 51 años o más & 14 & 19.2 \\
\hline & Total & 73 & 100.0 \\
\hline \multirow{5}{*}{ Años de experiencia docente } & Menos de 5 años & 18 & 24.7 \\
\hline & Entre 5 y 10 años & 15 & 20.5 \\
\hline & Entre 11 y 20 años & 16 & 21.9 \\
\hline & Más de 20 años & 24 & 32.9 \\
\hline & Total & 73 & 100.0 \\
\hline \multirow{3}{*}{$\begin{array}{c}\text { Ubicación de la institución } \\
\text { educativa }\end{array}$} & Urbana & 65 & 89.0 \\
\hline & Rural & 8 & 11.0 \\
\hline & Total & 73 & 100.0 \\
\hline
\end{tabular}

Validación de la escala

Debido a que el cuestionario implementado en esta investigación ha sido generado a partir de los resultados de la investigación adelantada por Fernández, Hernández, Prada y Ramírez (2020), del trabajo de Danielson (2013) y de los documentos emanados por la NCTM (2020) y el Ministerio de Educación Nacional de Colombia (2006); se requiere de realizar el proceso de validación del mismo, puesto que en muchos casos la redacción de los ítems fue cambiada de la versión original.

Apoyado en la teoría clásica de los test, se pretende garantizar que el instrumento mida lo que se desea medir cumpliendo dos condiciones: a) mínimo error y b) mayor precisión. Para evaluar la calidad del contenido se recurrió al juicio de expertos, para lo cual se conformó un grupo de seis personas integrado por los tres investigadores, dos docentes de Matemáticas en ejercicio en los niveles de Básica y Media, y un docente con rol de Coordinador académico. Tras varias rondas de revisión se ajustó la redacción de cada uno de los ítems y se procedió a aplicar el cuestionario a este grupo de docentes.

Para evaluar la fiabilidad del cuestionario se analizó el índice de discriminación - Id, "dado que los ítems del instrumento corresponden a variables no cognitivas, el principal indicador para evaluar el comportamiento individual de cada ítem y su contribución a cada subescala y la escala global es su capacidad discriminativa" (Fernández, Hernández, Prada y Ramírez, 
2020, p. 1190). De acuerdo con Muñiz et al. (2005) un ítem discrimina bien si su Id es de por lo menos .30. Al realizar una revisión inicial de la totalidad de los ítems (125) considerados en el cuestionario, se identificó que 30 de ellos presentaban un valor de Id inferior a .30, razón por la cual si se excluían del cuestionario ello traería como efecto un aumento en el coeficiente de confiabilidad Alfa de Cronbach. A partir de esta exploración inicial se determinan los descriptivos de los ítems que resultaron significativos para la escala en estudio y se muestran por medio de las Tablas 3 y 4.

Tabla 3. Estadísticas de fiabilidad

\begin{tabular}{cc}
\hline Alfa de Cronbach & N de elementos \\
\hline 0.974 & 95 \\
\hline
\end{tabular}

A partir del valor del Alfa de Cronbach obtenido en la Tabla 3 y asumiendo el criterio definido por Montgomery y Runger (2010), se concluye que la consistencia interna del cuestionario es adecuada. Respecto a los valores mostrados por medio de la Tabla 4, se puede concluir que todos los 95 ítems considerados para la construcción de la escala, discriminan muy bien y ello se ve reflejado en que, si se suprime cualquiera de ellos de la escala, el valor del coeficiente Alfa no mejoraría en nada.

Tabla 4. Estadísticas de total de elemento

\begin{tabular}{|c|c|c|c|c|c|}
\hline $\begin{array}{l}\text { Codificación } \\
\text { del Ítem }\end{array}$ & $\begin{array}{l}\text { Correlación } \\
\text { total de } \\
\text { elementos } \\
\text { corregida - Id }\end{array}$ & $\begin{array}{c}\text { Alfa de } \\
\text { Cronbach si } \\
\text { el elemento } \\
\text { se ha } \\
\text { suprimido }\end{array}$ & $\begin{array}{l}\text { Codificación } \\
\text { del Ítem }\end{array}$ & $\begin{array}{l}\text { Correlación } \\
\text { total de } \\
\text { elementos } \\
\text { corregida } \\
\text { - Id }\end{array}$ & $\begin{array}{c}\text { Alfa de } \\
\text { Cronbach si } \\
\text { el elemento } \\
\text { se ha } \\
\text { suprimido }\end{array}$ \\
\hline $\mathrm{C} 8$ & 0.532 & 0.974 & Represt5 & 0.572 & 0.974 \\
\hline $\mathrm{C9}$ & 0.503 & 0.974 & Represt6 & 0.453 & 0.974 \\
\hline C12 & 0.431 & 0.974 & Represt7 & 0.557 & 0.974 \\
\hline $\mathrm{C} 13$ & 0.375 & 0.974 & Mold1 & 0.436 & 0.974 \\
\hline $\mathrm{A} 1$ & 0.456 & 0.974 & Mold2 & 0.779 & 0.974 \\
\hline A3 & 0.538 & 0.974 & Mold4 & 0.395 & 0.974 \\
\hline A4 & 0.495 & 0.974 & Mold5 & 0.520 & 0.974 \\
\hline A5 & 0.438 & 0.974 & Mold6 & 0.517 & 0.974 \\
\hline A6 & 0.545 & 0.974 & Mold8 & 0.445 & 0.974 \\
\hline A7 & 0.490 & 0.974 & Conx1 & 0.487 & 0.974 \\
\hline A8 & 0.493 & 0.974 & Conx2 & 0.388 & 0.974 \\
\hline A9 & 0.442 & 0.974 & Conx3 & 0.490 & 0.974 \\
\hline A15 & 0.427 & 0.974 & Conx4 & 0.469 & 0.974 \\
\hline E2 & 0.307 & 0.974 & Conx5 & 0.459 & 0.974 \\
\hline E8 & 0.405 & 0.974 & Conx6 & 0.527 & 0.974 \\
\hline Crea1 & 0.497 & 0.974 & Conx7 & 0.564 & 0.974 \\
\hline Crea2 & 0.489 & 0.974 & Conx8 & 0.329 & 0.974 \\
\hline Crea3 & 0.359 & 0.974 & Plant2 & 0.384 & 0.974 \\
\hline
\end{tabular}




\begin{tabular}{|c|c|c|c|c|c|}
\hline Form1 & 0.577 & 0.974 & Plant3 & 0.441 & 0.974 \\
\hline Form2 & 0.593 & 0.974 & Plant4 & 0.668 & 0.974 \\
\hline Form3 & 0.484 & 0.974 & Plant5 & 0.592 & 0.974 \\
\hline Form4 & 0.365 & 0.974 & Plant6 & 0.566 & 0.974 \\
\hline Form5 & 0.664 & 0.974 & Plant7 & 0.644 & 0.974 \\
\hline Form6 & 0.714 & 0.974 & Plant8 & 0.431 & 0.974 \\
\hline Form7 & 0.566 & 0.974 & Plant9 & 0.605 & 0.974 \\
\hline Form8 & 0.667 & 0.974 & Plant10 & 0.497 & 0.974 \\
\hline Form9 & 0.671 & 0.974 & Plant11 & 0.372 & 0.974 \\
\hline Razon1 & 0.507 & 0.974 & Amb1 & 0.529 & 0.974 \\
\hline Razon2 & 0.661 & 0.974 & Amb2 & 0.557 & 0.974 \\
\hline Razon3 & 0.412 & 0.974 & Amb3 & 0.666 & 0.974 \\
\hline Razon4 & 0.637 & 0.974 & Amb4 & 0.546 & 0.974 \\
\hline Razon5 & 0.606 & 0.974 & Amb5 & 0.446 & 0.974 \\
\hline Razon6 & 0.641 & 0.974 & Amb6 & 0.621 & 0.974 \\
\hline Razon7 & 0.662 & 0.974 & Amb7 & 0.636 & 0.974 \\
\hline Razon8 & 0.728 & 0.974 & Amb8 & 0.681 & 0.974 \\
\hline Comun1 & 0.590 & 0.974 & PP2 & 0.512 & 0.974 \\
\hline Comun2 & 0.555 & 0.974 & PP3 & 0.534 & 0.974 \\
\hline Comun3 & 0.515 & 0.974 & PP4 & 0.606 & 0.974 \\
\hline Comun4 & 0.462 & 0.974 & PP6 & 0.630 & 0.974 \\
\hline Comun5 & 0.557 & 0.974 & PP7 & 0.622 & 0.974 \\
\hline Comun6 & 0.542 & 0.974 & PP8 & 0.556 & 0.974 \\
\hline Comun7 & 0.596 & 0.974 & PP9 & 0.606 & 0.974 \\
\hline Comun8 & 0.760 & 0.974 & PP10 & 0.685 & 0.974 \\
\hline Comun9 & 0.511 & 0.974 & PP11 & 0.479 & 0.974 \\
\hline Represt1 & 0.687 & 0.974 & PP13 & 0.558 & 0.974 \\
\hline Represt2 & 0.643 & 0.974 & PP14 & 0.541 & 0.974 \\
\hline Represt3 & 0.693 & 0.974 & PP15 & 0.515 & 0.974 \\
\hline Represt4 & 0.576 & 0.974 & & & \\
\hline
\end{tabular}

Nota: En caso de que el lector esté interesado en la redacción de los ítems se puede comunicar con los autores para facilitar copia del cuestionario utilizado.

\section{Perfiles de conocimiento didáctico- matemático de los docentes}

Debido a que este informe corresponde a los resultados de una prueba piloto se ha desconocido la recomendación sugerida por Hair et al. (2010) en lo que respecta al número de informantes necesario para poder cumplir con las exigencias de la aplicación del Análisis Factorial Exploratorio - AFE.

Al ingresar los 95 ítems y solicitar al software la reducción de factores, el software los agrupa en 23 factores todos con autovalores mayores a uno, con comunalidades que oscilan entre .70 y .95, al tiempo que logran explicar el $84.39 \%$ 
de la varianza total. Debido al amplio número de factores generados, se inicia un proceso de reducción de factores hasta alcanzar un menor número de ellos pero que no afecte tanto los parámetros.

En ese sentido, se llega a una propuesta de diez factores los cuales ofrecen comunalidades que oscilan entre .480 y .792 , al tiempo que logran explicar el $64.62 \%$ de la varianza acumulada.

A continuación, se realiza el análisis de cada uno de los componentes o factores latentes en que se agrupan los ítems del cuestionario, a la luz de los referentes o posibles categorías para la identificación de los perfiles docentes mencionados en la introducción.

\begin{tabular}{|l|c|}
\hline \multicolumn{2}{|c|}{ Tabla 5. Características del Análisis Factorial - Factor 1 } \\
\hline \multicolumn{1}{|c|}{ Ítems } & Carga \\
\hline $\begin{array}{l}\text { Amb2. Hace del salón de clase un espacio donde se valora el trabajo del docente y } \\
\text { de los estudiantes. }\end{array}$ & 0.744 \\
\hline Amb5. Monitorea los procedimientos de la clase y el uso eficiente del tiempo. & 0.697 \\
\hline $\begin{array}{l}\text { Amb1. Fomenta en el salón de clase el respeto y el trato amable entre todas las } \\
\text { personas. }\end{array}$ & 0.689 \\
\hline $\begin{array}{l}\text { Amb7. Establece normas de convivencia en el aula de clase yasigna responsabilidades } \\
\text { a sus estudiantes. }\end{array}$ & 0.649 \\
\hline $\begin{array}{l}\text { Plant8. Su planeación curricular se fundamenta en los estándares básicos de } \\
\text { competencias en matemáticas y se articula con el PEl de la institución. }\end{array}$ & 0.643 \\
\hline $\begin{array}{l}\text { Amb8. Hace uso del entorno físico de la institución para promover el aprendizaje de } \\
\text { los estudiantes. }\end{array}$ & 0.617 \\
\hline $\begin{array}{l}\text { Amb3. Motiva a los estudiantes a expresar sus puntos de vista por medio del uso del } \\
\text { lenguaje adecuado. }\end{array}$ & 0.603 \\
\hline $\begin{array}{l}\text { Amb6. Establece la creación de grupos de trabajo para la realización del aprendizaje } \\
\text { cooperativo en el desarrollo de sus clases. }\end{array}$ & 0.552 \\
\hline $\begin{array}{l}\text { PP7. Las preguntas que presenta a sus estudiantes, invitan a formular hipótesis, } \\
\text { hacer conexiones o a desafiar ideas previas. }\end{array}$ & 0.489 \\
\hline Amb4. Establece las rutinas de clase que permiten el buen funcionamiento de esta. & 0.481 \\
\hline $\begin{array}{l}\text { Plant9. Su planeación curricular refleja la secuencia de procesos de aprendizaje } \\
\text { garantizando el avance de los estudiantes }\end{array}$ & 0.450 \\
\hline
\end{tabular}

El Factor 1 explica el $31.54 \%$ de la varianza total. Según las características de los ítems agrupados en él, se podría reconocer como el perfil del docente en el que se conjuga el conocimiento pedagógico general con el conocimiento curricular de la asignatura en términos de Shulman (1987). Es un docente que refleja fuerte experiencia en el componente pedagógico pues evidencia manejo de aula con conocimiento del marco normativo que regula su trabajo disciplinar. 
Tabla 6. Características del Análisis Factorial - Factor 2

\begin{tabular}{|c|c|}
\hline Ítems & Carga \\
\hline $\begin{array}{l}\text { Represt3. Utiliza modelos ejemplificadores (esquemas, dibujos del proceso o } \\
\text { situación, entre otros) para mostrar maneras de resolver situaciones problemáticas. }\end{array}$ & 0.694 \\
\hline $\begin{array}{l}\text { Represt1. Pide al estudiante que hable, escuche y reflexione sobre las matemáticas } \\
\text { para avanzar hacia la utilización de símbolos propios del lenguaje formal matemático. }\end{array}$ & 0.668 \\
\hline Razon6. Promueve el apoyo del razonamiento matemático. & 0.659 \\
\hline $\begin{array}{l}\text { Conx1. Considera las experiencias cotidianas de los estudiantes para avanzar hacia } \\
\text { las matemáticas más formales. }\end{array}$ & 0.611 \\
\hline $\begin{array}{l}\text { Razon8. Propone a los estudiantes posibles respuestas con el fin de que ellos las } \\
\text { adopten o rechacen, pero con los respectivos argumentos. }\end{array}$ & 0.571 \\
\hline $\begin{array}{l}\text { Mold2. Utiliza modelos para comprender una idea o concepto con el fin de facilitar } \\
\text { su manejo. }\end{array}$ & 0.564 \\
\hline $\begin{array}{l}\text { Plant7. En su práctica pedagógica incorpora diversos recursos disponibles fuera de } \\
\text { la institución con el fin de garantizar el aprendizaje del estudiante. }\end{array}$ & 0.547 \\
\hline Razon7. Realiza retroalimentación con material concreto manipulativo. & 0.516 \\
\hline A9. En mi familia, hay personas a las cuales les gusta las matemáticas. & 0.450 \\
\hline $\begin{array}{l}\text { Comun7. Interviene mayoritariamente a través de preguntas, más que a través de } \\
\text { explicaciones }\end{array}$ & 0.419 \\
\hline Comun8. Utiliza diversas formas para expresar y representar un contenido matemático & 0.410 \\
\hline $\begin{array}{l}\text { Form4. Realiza preguntas que propician la investigación y exploración para solucionar } \\
\text { un problema matemático }\end{array}$ & 0.405 \\
\hline $\begin{array}{l}\text { Mold8. Su objetivo es que el estudiante construya modelos matemáticos acompañados } \\
\text { de la respectiva argumentación }\end{array}$ & 0.403 \\
\hline
\end{tabular}

El Factor 2 explica el 5.52\% de la varianza total. A partir de las características de los ítems agrupados se identifica el perfil de un docente con conocimiento pedagógico del contenido como lo afirma Shulman (1987). Siendo un docente competente en la enseñanza propia de su disciplina, que se apoya de diversos recursos didácticos como representaciones semióticas y sus articulaciones coherentes, acompañado de la formulación de preguntas para facilitar la comprensión de situaciones y el razonamiento de las mismas en busca de posibles alternativas de solución. Corresponde a las características ideales de un buen docente de matemáticas.

\begin{tabular}{|l|c|}
\hline \multicolumn{2}{|c|}{ Tabla 7. Características del Análisis Factorial - Factor 3 } \\
\hline \multicolumn{1}{|c|}{ Ítems } & Carga \\
\hline A5. Mi relación con los estudiantes es de mucho respeto y cordialidad. & 0.778 \\
\hline A7. Me intereso por la evolución y rendimiento de los estudiantes en mi asignatura. & 0.778 \\
\hline $\begin{array}{l}\text { A4. Siempre estoy dispuesto a aclarar las dudas y dificultades que surjan a mis } \\
\text { estudiantes durante la clase. }\end{array}$ & 0.647 \\
\hline $\begin{array}{l}\text { A6. Los profesores que explican con bastante claridad y entusiasmo hacen que sus } \\
\text { estudiantes gusten de las matemáticas. }\end{array}$ & 0.646 \\
\hline C8. Busco distintas maneras y métodos para resolver un problema. & 0.621 \\
\hline A8. Valoro el esfuerzo y reconozco el trabajo diario de los estudiantes en mi asignatura. & 0.589 \\
\hline
\end{tabular}




\begin{tabular}{|l|c|}
\hline $\begin{array}{l}\text { PP15. En el proceso de seguimiento del aprendizaje de los estudiantes, recurre a la } \\
\text { retro alimentación para hacer correcciones cuando es necesario. }\end{array}$ & 0.545 \\
\hline $\begin{array}{l}\text { A3. En clase de matemáticas empleo gran variedad de medios y ejemplos prácticos } \\
\text { que permiten relacionar las matemáticas con situaciones de la vida diaria. }\end{array}$ & 0.520 \\
\hline $\begin{array}{l}\text { PP4. Cuando en la clase debo ampliar las explicaciones, utilizo ejemplos relacionados } \\
\text { con los intereses de los estudiantes o con temas vistos con anterioridad. }\end{array}$ & 0.499 \\
\hline Crea1. Me considero creativo en mi trabajo y en mi vida personal. & 0.434 \\
\hline
\end{tabular}

El Factor 3 explica el $5.40 \%$ de la varianza total. A partir de las características de los ítems agrupados se identifica el perfil de un docente cuyo sistema de creencias está orientado al conocimiento de los estudiantes y sus características en términos de Shulman (1987). Este docente se inclina por el reconocimiento del estudiante como un ser humano que es movido por sus sentimientos y emociones.

\begin{tabular}{|c|c|}
\hline Ítems & Carga \\
\hline $\begin{array}{l}\text { Form3. Propone situaciones problemáticas de diversos tipos sobre el mismo concepto } \\
\text { matemático. }\end{array}$ & 0.757 \\
\hline $\begin{array}{l}\text { Represt6. Trabaja en los estudiantes las representaciones simbólicas (notación } \\
\text { matemática convencional, como fórmulas, entre otros). }\end{array}$ & 0.745 \\
\hline $\begin{array}{l}\text { Mold4. Recurre a la formulación de preguntas, para que el estudiante entienda el } \\
\text { contexto del problema y así facilitar el proceso de modelado. }\end{array}$ & 0.717 \\
\hline $\begin{array}{l}\text { Comun9. Promueve en el aula la articulación coherente de diversos registros de } \\
\text { representación alrededor de los conceptos matemáticos. }\end{array}$ & 0.703 \\
\hline $\begin{array}{l}\text { Comun4. Pide a estudiantes explicar con el lenguaje matemático adecuado a su } \\
\text { nivel, sus estrategias y respuestas. }\end{array}$ & 0.699 \\
\hline $\begin{array}{l}\text { Comun1. Promueve con mayor énfasis el intercambio de ideas en el aula, que la } \\
\text { entrega de información unidireccional por su parte. }\end{array}$ & 0.691 \\
\hline $\begin{array}{l}\text { Mold1. Utiliza la modelación matemática para representar la realidad en forma } \\
\text { esquemática. }\end{array}$ & 0.670 \\
\hline $\begin{array}{l}\text { Represt7. En su trabajo de aula, pasa de lo concreto a lo abstracto, y volviendo de lo } \\
\text { abstracto a lo concreto. }\end{array}$ & 0.664 \\
\hline $\begin{array}{l}\text { Plant2. Tiene identificados los conceptos que casi siempre son comprendidos de } \\
\text { manera equivocada por los estudiantes y con su planeación pretenden corregirlos. }\end{array}$ & 0.588 \\
\hline A15. Dominar las matemáticas me permitirá tener éxito en mis estudios posgraduales. & 0.556 \\
\hline
\end{tabular}

El Factor 4 explica el $4.37 \%$ de la varianza total. A partir de las características de los ítems agrupados se identifica el perfil de un docente que está orientado al conocimiento del contenido en términos como lo señala Shulman (1987). Este docente reconoce la naturaleza abstracta del saber matemático y por ello en su práctica pedagógica hace especial énfasis en aquellas actividades centradas en la comunicación, tal como lo afirma Duval (2012) "el acceso a los objetos matemáticos se hace únicamente por medio de la producción de representaciones semióticas, y no por la percepción o la utilización de instrumentos como ocurre en las otras ciencias" (p. 15). 
Tabla 9. Características del Análisis Factorial - Factor 5

\begin{tabular}{|l|c|}
\hline \multicolumn{2}{|c|}{ Tabla 9. Características del Análisis Factorial - Factor 5 } \\
\hline \multicolumn{1}{|c|}{ Ítems } & Carga \\
\hline $\begin{array}{l}\text { Conx6. Genera conocimiento matemático a través de contextos vinculados a la } \\
\text { psicomotricidad. }\end{array}$ & 0.777 \\
\hline Conx3. Desarrolla actividades matemáticas vinculadas a contextos musicales. & 0.704 \\
\hline Conx4. Trabaja las matemáticas vinculándolas con la literatura infantil. & 0.702 \\
\hline Conx5. Relaciona las matemáticas con la expresión artística. & 0.683 \\
\hline $\begin{array}{l}\text { Plant3. Al momento de diseñar actividades de aprendizaje, considera los diversos } \\
\text { ritmos de aprendizaje de sus estudiantes. }\end{array}$ & 0.537 \\
\hline $\begin{array}{l}\text { Represt2. Utiliza materiales concretos como recursos didácticos para representar } \\
\text { ideas matemáticas. }\end{array}$ & 0.512 \\
\hline $\begin{array}{l}\text { Plant4. Al momento de diseñar las actividades de aprendizaje, considera las } \\
\text { necesidades particulares de sus estudiantes. }\end{array}$ & 0.483 \\
\hline $\begin{array}{l}\text { PP13. Observa lo que los estudiantes escriben o las preguntas planteadas por ellos, } \\
\text { con el fin de evaluar si es necesario hacer más actividades o ampliar las explicaciones. }\end{array}$ & 0.472 \\
\hline $\begin{array}{l}\text { Form5. Permite al estudiante usar material concreto y/o pictórico con apoyo oral para } \\
\text { que trabajen en la resolución de problemas matemáticos. }\end{array}$ & 0.401 \\
\hline
\end{tabular}

El Factor 5 explica el $3.48 \%$ de la varianza total. A partir de las características de los ítems agrupados en él, se identifica el perfil de un docente que está orientado al conocimiento del contexto (Grossman, 1990). Este docente reconoce como elemento importante de su práctica pedagógica en la enseñanza de las
Matemáticas, el establecimiento de conexiones de los conceptos con las diversas actividades que realiza el estudiante en su cotidianidad, en ese ejercicio pedagógico se reconocen las particularidades del estudiante y del contexto en

\begin{tabular}{|l|c|}
\hline \multicolumn{2}{|c|}{ Tabla 10. Características del Análisis Factorial - Factor 6 } \\
\hline \multicolumn{1}{|c|}{ Ítems } & Carga \\
\hline $\begin{array}{l}\text { PP8. Impulsa a sus estudiantes a participar haciendo uso del lenguaje preciso para } \\
\text { profundizar en la comprensión de conceptos. }\end{array}$ & 0.816 \\
\hline $\begin{array}{l}\text { Conx8. Promueve que los estudiantes apliquen el conocimiento matemático en el } \\
\text { contexto de la naturaleza y en la preservación del medio ambiente. }\end{array}$ & 0.614 \\
\hline $\begin{array}{l}\text { PP11. Propone actividades organizadas de forma que proporciona desafíos cognitivos } \\
\text { y buscan animar a los estudiantes a reflexionar sobre lo que han hecho o lo que han } \\
\text { aprendido. }\end{array}$ & 0.579 \\
\hline $\begin{array}{l}\text { PP9. Motiva a sus estudiantes a la formulación de preguntas de forma individual o } \\
\text { grupal, como recurso de apoyo en el aula. }\end{array}$ & 0.575 \\
\hline $\begin{array}{l}\text { PP6. Hace uso de la indagación y la discusión como técnicas para profundizar la } \\
\text { comprensión de estudiante. }\end{array}$ & 0.555 \\
\hline $\begin{array}{l}\text { PP10. Las actividades que presenta en el aula, les permiten a los estudiantes hacer } \\
\text { contribuciones a la vida intelectual de la clase. }\end{array}$ & 0.529 \\
\hline $\begin{array}{l}\text { Plant6. En su práctica pedagógica hace uso de los recursos más adecuados } \\
\text { disponibles en la institución con el fin de garantizar el aprendizaje del estudiante. }\end{array}$ & 0.454 \\
\hline
\end{tabular}




\begin{tabular}{|l|c|}
\hline $\begin{array}{l}\text { Form9. Permite que los estudiantes inventen sus propios problemas y los motiva a } \\
\text { resolverlos en la clase. }\end{array}$ & 0.451 \\
\hline $\begin{array}{l}\text { Form8. Propone situaciones en las que le sobra o le falta información, para que los } \\
\text { estudiantes deban proponer las preguntas. }\end{array}$ & 0.441 \\
\hline $\begin{array}{l}\text { PP14. Les enseña a sus estudiantes las habilidades necesarias de seguimiento de su } \\
\text { propio aprendizaje frente a unas metas claras. }\end{array}$ & 0.411 \\
\hline $\begin{array}{l}\text { Form7. Promueve la discusión en torno a las estrategias de resolución de problemas } \\
\text { y la validez de los resultados obtenidos. }\end{array}$ & 0.401 \\
\hline
\end{tabular}

El Factor 6 explica el $3.33 \%$ de la varianza total. A partir de las características de los ítems agrupados en él, se identifica el perfil de un docente que está orientado al conocimiento pedagógico del contexto específicamente en la segunda categoría de este conocimiento que define Grossman (1990) como el conocimiento de la comprensión de los estudiantes. Este docente centra su atención en el estudiante, en el conocimiento de sus concepciones correctas e incorrectas para a partir de ellas implementar actividades y recursos bien sea para fortalecer los aciertos o aclarar los obstáculos epistemológicos que tienen.

\begin{tabular}{|l|c|}
\hline \multicolumn{2}{|c|}{ Tabla 11. Características del Análisis Factorial - Factor 7 } \\
\hline \multicolumn{1}{|c|}{ Ítems } & Carga \\
\hline Conx2. Realiza conexiones entre diversos contenidos matemáticos. & 0.665 \\
\hline $\begin{array}{l}\text { Razon3. Pide a los estudiantes que expliquen, justifiquen o argumenten las estrategias } \\
\text { o técnicas que utilizaron durante la resolución de un problema matemático. }\end{array}$ & 0.588 \\
\hline $\begin{array}{l}\text { Razon2. Permite que los estudiantes descubran, analicen y propongan diversas vías } \\
\text { de resolución. }\end{array}$ & 0.577 \\
\hline $\begin{array}{l}\text { Razon4. Plantea interrogantes para ayudar a que los estudiantes argumenten sus } \\
\text { respuestas. }\end{array}$ & 0.567 \\
\hline Razon5. Promueve que los estudiantes comprueben conjeturas de la vida cotidiana. & 0.534 \\
\hline $\begin{array}{l}\text { PP2. Presenta a sus estudiantes de forma clara los objetivos de la clase y las } \\
\text { instrucciones de cada una de las actividades a realizar. }\end{array}$ & 0.514 \\
\hline Comun6. Fomenta la escucha atenta de los puntos de vista de los demás. & 0.499 \\
\hline $\begin{array}{l}\text { PP3. Presenta los conceptos con precisión, claridad e imaginación utilizando el } \\
\text { lenguaje académico adecuado. }\end{array}$ & 0.462 \\
\hline $\begin{array}{l}\text { Form6. Mantiene al estudiante comprometido y participando activamente en el } \\
\text { proceso de resolución de problemas matemáticos. }\end{array}$ & 0.418 \\
\hline
\end{tabular}

El Factor 7 explica el $3.23 \%$ de la varianza total. A partir de las características de los ítems agrupados en él, se identifica el perfil de un docente que está orientado al conocimiento especializado del contenido argumentado por Ball, Thames y Phelps (2008). Este docente centra su proceso de enseñanza en la presentación exacta de los conceptos matemáticos, proporcionando explicaciones sobre los recursos disponibles a utilizar con el fin que el estudiante razone, analice y especule sobre las diferentes alternativas de solución ante los diversos problemas propuestos.

Las características de los tres factores restantes se muestran por medio de la Tabla 12 observando que sus porcentajes de varianza explicada son 
inferiores al $3 \%$, pero a pesar de ello permite identificar tres perfiles de docentes más de los ya caracterizados.

Tabla 12. Descripción de los Factores restantes del análisis factorial

\begin{tabular}{cccc}
\hline Factor & $\begin{array}{c}\mathbf{N}^{\circ} \text { de ítems } \\
\text { que agrupa }\end{array}$ & $\begin{array}{c}\text { \% Varianza } \\
\text { explicada }\end{array}$ & \multicolumn{1}{c}{ Descripción } \\
\hline 8 & 7 & $2.84 \%$ & $\begin{array}{l}\text { Este docente motiva a sus estudiantes a sentir curiosidad } \\
\text { por la solución a situaciones propuestas, pero les da } \\
\text { libertades a que ellos exploren alternativas de solución, } \\
\text { utilizando como método el ensayo y el error, pero todo } \\
\text { dentro de un contexto de aprendizaje cooperativo. }\end{array}$ \\
\hline 9 & 5 & $2.61 \%$ & $\begin{array}{l}\text { Este docente centra su actuación pedagógica en la } \\
\text { evaluación y en la importancia que tienen los resultados } \\
\text { de la misma sobre el proceso de aprendizaje. }\end{array}$ \\
\hline 10 & 3 & $\begin{array}{l}\text { El docente de este factor se caracteriza por tener una } \\
\text { fuerte autoestima sobre sus habilidades en matemáticas, } \\
\text { por ende, tiene la creencia que ello lo hace un buen } \\
\text { docente. }\end{array}$ \\
\hline
\end{tabular}

\section{CONCLUSIONES}

Tras la realización de esta investigación se puede tener una aproximación a lo complejo que resulta caracterizar el conocimiento matemático que poseen los docentes que hoy día orientan esta asignatura en las aulas de clase.

Es claro que a partir de la concepción que los docentes tienen sobre lo que es el conocimiento matemático, orientan todo su actuar pedagógico en el que se ubica la preparación de las clases, las actividades que se desarrollan dentro o fuera del aula, los recursos didácticos que implementa en el proceso de enseñanza y la evaluación en sí misma que usará para determinar si el estudiante alcanzó sus metas de aprendizaje.

Se sugiere que este trabajo sea replicado con una muestra mayor de 400 docentes para contrastar los hallazgos y de esta manera dar una fiabilidad más amplia del cuestionario diseñado y las categorías de perfiles docentes identificadas.

\section{REFERENCIAS BIBLIOGRÁFICAS}

Arce, A. L. A., Alpízar, M., López, Y. M., formación inicial y continua de docentes de matemáticas en Costa Rica. Cuadernos de Investigación y formación en Educación Matemática, 131-173.

Ball, D. L., Thames, M. H., \& Phelps, G. (2008). Content knowledge for teaching. What makes it special? Journal of Teacher Education, 59(5), 389-407.

Danielson, C. (2013). The Framework for Teaching. Association for Supervision and Curriculum Development.

Duval, R. (2012). Lo esencial de los procesos cognitivos de comprensión en matemáticas: los registros de representación semiótica. Resúmenes del VI Coloquio Internacional de Didáctica de las Matemáticas: avances y desafíos actuales, 14-17.

Echeverría, S., Martínez, S., Carmona, M., Alamilla, J. C., Benítez, J. E., \& Velázquez, J. H. (2017). Una formación docente acorde 
a los cambios generacionales, tecnológicos e innovadores en los programas de postgrado nacionales. Temas de Ciencia y Tecnología, 21(63), 33-38.

Escobar Medina, M. B. (2015). Influencia de la interacción alumno-docente en el proceso enseñanza-aprendizaje. Paakat: Revista de Tecnología y Sociedad, (8).

Fernández-Cézar, R., Hernández-Suárez, C. A., Prada-Núñez, R., \& Ramírez-Leal, P. (2020). Creencias y ansiedad hacia las matemáticas: un estudio comparativo entre maestros de Colombia y España. Bolema: Boletim de Educação Matemática, 34(68), 1174-1205.

Gamboa Araya, R. (2014). Relación entre la dimensión afectiva y el aprendizaje de las matemáticas. Revista electrónica educare, 18(2), 117-139.

Gamboa Araya, R. (2016). ¿Es necesario profundizar en la relación entre docente de matemáticas y la formación de las actitudes y creencias hacia la disciplina? Uniciencia, 30(1), 57-84.

Gómez-Chacón, I. M., Op’t Eynde, P., \& De Corte, E. (2006). Creencias de los estudiantes de matemáticas. La influencia del contexto de clase. Enseñanza de las ciencias: revista de investigación y experiencias didácticas, 309-324.

Grossman, P. (1990). The making of a teacher: Teacher knowledge and teacher education. New York and London: Teachers College Press.

Hill, H. C., Ball, D. L., \& Schlling, S. G. (2008). Unpacking pedagogical content knowledge of students. Journal for Research in Mathematics Education, 39, 372-400.

Márquez, Rosa María Farfán, \& Ramos, María Guadalupe Simón. (2018). El Desarrollo del Talento de las Mujeres en Matemáticas desde la Socioepistemología y la Perspectiva de Género: un Estudio de Biografías. Bolema: Boletim de Educação Matemática, 32(62), 946-966. https://doi. org/10.1590/1980-4415v32n62a10.

Mayorca, V. M., Camacho, M. C., Trujillo, E. R., \& Artunduaga, L. C. (2009). Influencia de los estilos de comunicación asertiva de los docentes en el aprendizaje escolar. Psicogente, 12(21), 78-95.

Ministerio de Educación Nacional. (2006). Estándares Básicos de Competencias en Matemáticas en Lenguaje, Matemáticas, Ciencias y Ciudadanas. Bogotá: MEN.

National Council of Teachers of Mathematics. (2000). Principles and standards for school mathematics. NCTM.

Nortes, R., \& Nortes, A. (2017). Agrado y utilidad de las matemáticas en la formación inicial de maestros de educación primaria. PNA, 12(1), 27-42.

Pino-Fan, L.R., \& Godino, J.D. (2015). Perspectiva ampliada del conocimiento didácticomatemático del profesor. Paradigma, 36(1), 87-109.

Shulman, L. S. (1986). Those who understand: Knowledge growth in teaching. Educational Researcher, 15(2), 4-14.

Shulman, L. S. (1987). Knowledge and teaching: Foundations of the new reform. Harvard Educational Review, 57(1), 1-22.

Zamora, G. H. (2009). Escritura académica y formación de maestros ¿por qué no acaban la tesis? Tiempo de educar, 10(19), 11-40. 